\title{
Modelling of Foam Spread on a Burning Liquid Fuel Surface
}

\author{
BROR PERSSON, ANDERS LÖNNERMARK, and HENRY PERSSON \\ SP Swedish National Testing and Research Institute, Fire Technology, \\ Borås, Sweden.
}

\begin{abstract}
Theoretical models have been formulated describing the spread of foam on burning liquid fuel surfaces in pool-like configurations. The main driving force of the foam spread is due to differences in the hydrostatic pressure within the foam while the dominating resisting force is due to viscous friction between the foam blanket and the fuel beneath. Account is taken to the influence of ordinary drainage, radiation-induced drainage, and evaporation.

A large number of experiments have been performed to generate input data and to generate data for verification of the theoretical models. A series of experiments with water as "fuel" in a $1 / 2$-symmetric pool has been used to determine friction coefficients. These coefficients have then been scaled and used for other fuels to calculate the foam spread in a number of different scenarios, both with and without fire. There is in general a good agreement between the calculated and the experimental results.
\end{abstract}

KEYWORDS: Fire fighting foam, storage tank fires, foam spread experiments, foam spread modelling, FOAMSPEX, drainage characteristics

\section{NOTATION}

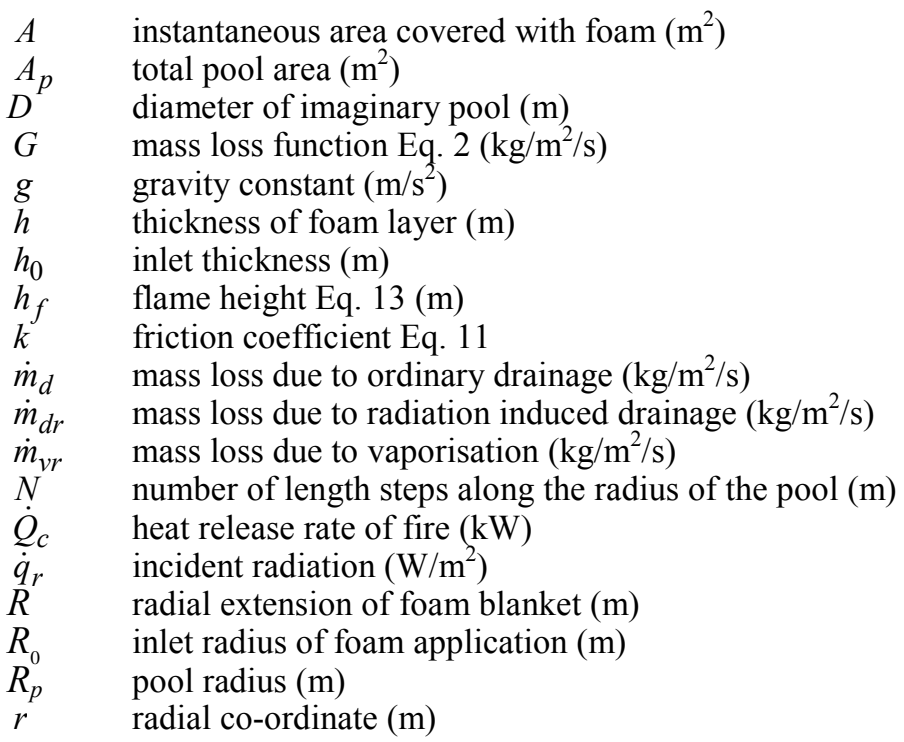




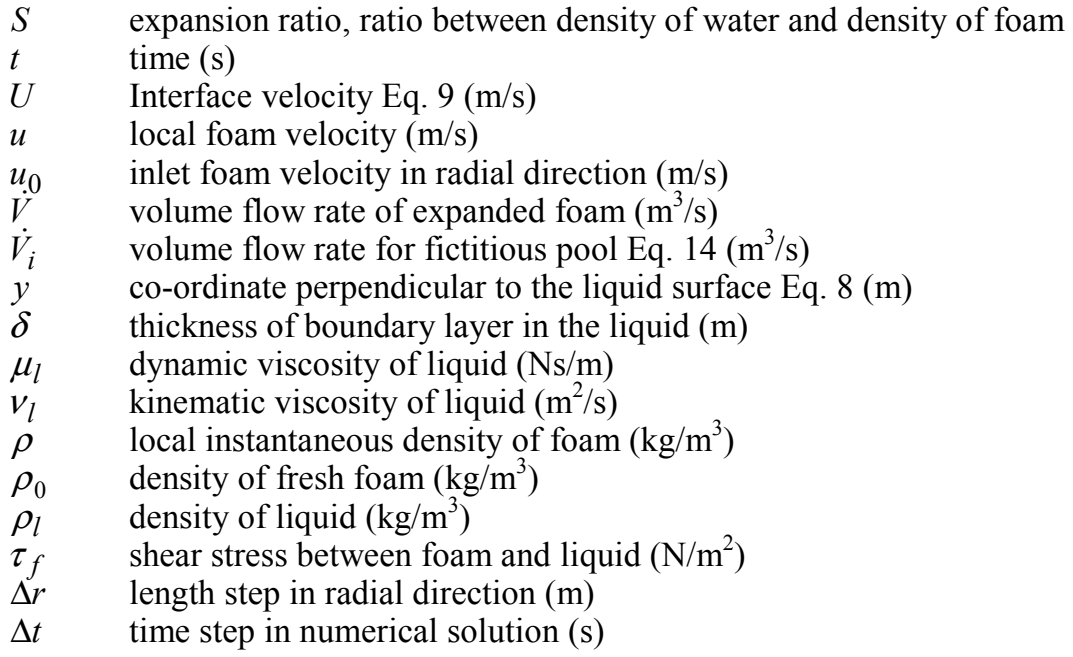

\section{INTRODUCTION}

Fully involved flammable liquid storage tank fires present many problems for fire fighters and in many cases extinguishing attempts have not been successful. Today's storage tanks can be more than $120 \mathrm{~m}$ in diameter. Fire fighting foam is the only practicable method for extinguishing large flammable liquid fires, but it is not fully understood if there is any upper practical limit for successful extinguishment. Fire protection standards like NFPA 11 only recognize foam monitors for use as primary protection for storage tanks up to $20 \mathrm{~m}$ in diameter. Even for fixed foam systems there are uncertainties whether recommended application rates and foam stocks are adequate.

In a recently completed EuropeanCommisssion-sponsored project, FOAMSPEX [1], a major step forward in understanding the extinguishment mechanisms and limitations of fire fighting foams has been taken. The topics covered in the project ranged from presentation of the fundamentals of foam spreading, which forms the basis for the models, through extensive experimental work to models, which predict foam spread and extinguishment of full-scale tanks up to $120 \mathrm{~m}$ in diameter. The experimental work, which has provided vital information for the development of the models, has included laboratory measurements of foam properties, foam spread tests in various geometries, field trials of foam monitors, and full scale validating fire tests.

The present paper focus on the theoretical models with gentle application (where the foam slides down gently onto the foam surface) developed in the FOAMSPEX project. Calculated results are compared to measurements from a number of experiments conducted to verify the models. In the FOAMSPEX project both circular geometries and channel configurations were studied, but in the present paper only the circular case is discussed. The same type of models has also been used for over-the-top application (direct application by using a foam nozzle directed towards the foam surface). For more details about the experimental work and the case with direct application the reader is referred to the report by Persson et al. [1]. 


\section{THEORETICAL MODEL}

In formulating the basic equations, the hydro dynamical approach is adopted, i.e. it is assumed that a driving force caused by hydrostatic pressure differences in the foam and a resisting friction force between the foam and the liquid governs the foam flow. Models for calculating the influence of ordinary drainage, radiation-induced drainage, and evaporation are given. A numerical solution scheme is outlined. More information about the theoretical model can be found in Persson et al. [1].

\section{Governing equations for central inlet}

Consider the spread of foam on a burning liquid fuel surface in a circular pool. The foam is assumed to be gently applied at an inlet radius $r=R_{0}$ with a constant volume flow rate $\dot{V}\left(\mathrm{~m}^{3} / \mathrm{s}\right)$ and advancing symmetrically in radial direction, see Fig. 1. The instantaneous thickness of the spreading foam layer is denoted by $h(r, t)$, the thickness at $r=R_{0}$ is $h_{0}$, and the instantaneous location of the foam front is given by $r=R(t)$. It is assumed that the viscosity of the foam is several orders of magnitude larger than the viscosity of water and ordinary hydrocarbon fuels, Kroezen et al. [2]. This means that the velocity gradients in the foam will be small compared to the gradients in the fluid and consequently the velocity in the foam layer can to a good approximation be assumed to be constant across the thickness. A more detailed discussion of the governing equations given below can be found in Persson et al. [3].

The continuity equation for the foam flow can be written

$$
\frac{\partial}{\partial t}(\rho r h)+\frac{\partial}{\partial r}(\rho r u h)=-r G
$$

Here $\rho$ denotes the local density of the foam layer, i.e. $\rho$ is generally a function of $r$ and $t$. The function $G=G(r, t)$ denotes the mass loss due to drainage and evaporation and can be expressed as

$$
G(r, t)=\dot{m}_{d}+\dot{m}_{d r}+\dot{m}_{v r}
$$

where $\dot{m}_{d}$ is the mass loss due to ordinary drainage, $\dot{m}_{d r}$ the additional drainage caused by radiation, and $\dot{m}_{v r}$ the mass loss due to vaporisation.

Assuming that the variation of the bulk density of the foam due to the mass loss can be expressed

$$
h\left(\frac{\partial \rho}{\partial t}+u \frac{\partial \rho}{\partial r}\right)=-G(r, t)
$$

makes it possible to derive a simplified continuity equation from Eq. 1, i.e.

$$
\frac{\partial}{\partial t}(r h)+\frac{\partial}{\partial r}(r u h)=0
$$




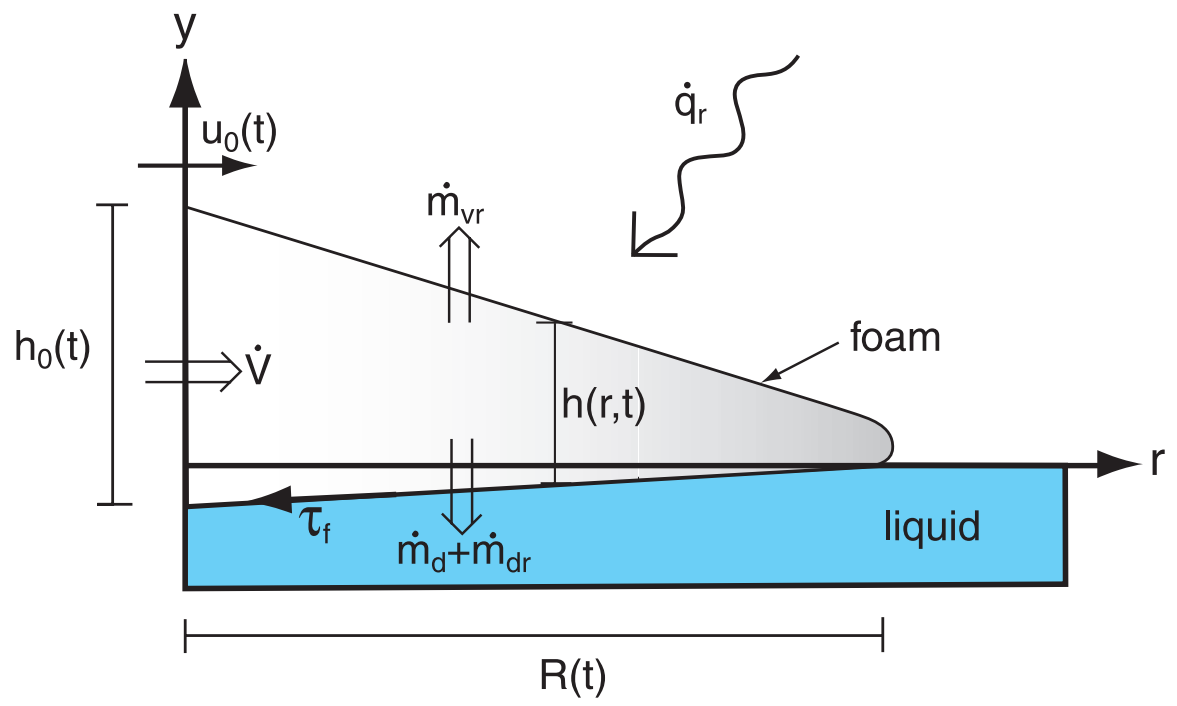

Fig. $1 \quad$ Schematic of a foam layer spreading on a liquid surface. The foam is applied from the left with a constant volume rate, $\dot{V}$, and a friction force, $\tau_{f}$, opposes the spread. The foam is exposed to a radiation flux, $\dot{q}_{r}$, causing an evaporation mass loss, $\dot{m}_{v r}$, and a radiation-induced drainage, $\dot{m}_{d r}$, in addition to the ordinary drainage, $\dot{m}_{d}$. The origin in the figure is located at the inlet radius at $r=R_{0}$.

A balance equation for the radial momentum takes the form

$$
\frac{\partial}{\partial t}(r u h)+\frac{\partial}{\partial r}\left(r u^{2} h\right)=-\frac{g}{2}\left(1-\frac{1}{S}\right) \frac{\partial}{\partial r}\left(r h^{2}\right)-\frac{r \tau_{f}}{\rho}
$$

Here the left hand side expresses the rate of change of inertia, the first term on the right hand side is the driving force caused by the hydrostatic pressure across the foam layer. The last term denotes the resulting friction force due to shear stress between foam and liquid. The parameter $S$ denotes the ratio between the density of the liquid $\rho_{l}$ and the density of the foam $\rho$. This parameter is called the expansion ratio and is normally used as one quality parameter of the foam. In this analysis the expansion ratio is treated as a constant. It is to be noted that the assumption of a constant expansion ratio does not imply that the foam density is constant.

Note that the influence of the drainage mass transport upon the momentum balance has been neglected. Equations (4) and (5) constitute the final set of equations describing the foam spread in a circular pool. At the inlet $\left(r=R_{0}\right)$ the density of the fresh foam $\rho_{0}$ is known, while the layer thickness $h_{0}$ and the inlet velocity $u_{0}$ are connected through the equation

$$
2 \pi R_{0} u_{0} h_{0}=\dot{V}
$$


An additional boundary condition is provided by the closure at the front of the foam layer, i.e.

$$
h(R, t)=0
$$

\section{Friction model}

When foam is applied at the centre of a circular pool the resistance to the foam spread will mainly emanate from the shear forces between the foam and the liquid. The flow set up in the pool can be considered as a backward boundary layer flow, i.e. a flow where the boundary layer thickness is increasing from the inlet towards the advancing front. At the foam front the flow field in the liquid cannot be described in terms of a boundary layer flow, therefore the resistance in the model is treated as a mean over the entire foam area rather than as a local entity.

The shear stress $\tau_{f}$ appearing in the momentum equation will be estimated by making use of solutions for boundary layer flow in the liquid. It is assumed that the viscosity of the foam is much larger than that of the liquid, which means that the shear deformation of the foam can be neglected. It is assumed that the liquid layer beneath the foam is deep enough to be considered as semi-infinite. Furthermore, the flow is assumed to be in a quasi steady state meaning that the time variation in the velocity field can be neglected.

An integral balance for the momentum change of the boundary layer in a liquid of infinite depth can be written

$$
\rho_{l} \frac{d}{d r} \int_{0}^{\delta} r u^{2} \cdot d y=r \tau_{f}
$$

where $\delta$ is the thickness of the boundary layer, $y$ is a co-ordinate perpendicular to the liquid surface (positive downwards), and $\tau_{f}$ is the shear stress at the interface between the foam and the liquid. By assuming a second order polynomial for the velocity profile and inserting into Eq. 8, an approximate solution of the boundary layer thickness $\delta$ can be derived

$$
\delta=\sqrt{\frac{20 v_{l} r}{3 U}}
$$

Here $v_{l}$ denotes the kinematic viscosity of the liquid and $U$ is the velocity at the interface. To simplify the calculations, the velocity has been assumed constant and the inlet radius has been set equal to zero. Upper and lower bounds for the velocity are $u_{0}$ (the inlet velocity) and $\dot{R}$ (the front velocity), i.e. $u_{0}>U>\dot{R}$. With aid of Eq. 9 and the assumed velocity profile the shear stress can be expressed

$$
\tau_{f}=\sqrt{\frac{3}{5}} \cdot \rho_{l} \sqrt{v_{l}} \cdot \frac{U^{3 / 2}}{r^{1 / 2}}
$$


A mean value of the shear stress over the foam area $\left(\bar{\tau}_{f}\right)$ can be defined by integrating Eq. 10. This analysis indicates that it would be conceivable to express the shear stress as

$$
\bar{\tau}_{f}=k \cdot \frac{u_{0}^{3 / 2}}{g(R)}
$$

where $k$ is a constant and $g(R)$ is a function of the extension. Comparison with experiments indicates a best fit of the data when assuming $g(R)=R^{1 / 4}$. Note that the friction coefficient $k$ in Eq. 11 depends on the type of fuel and is proportional to the density and the square root of the kinematic viscosity according to Eq. 10. Thus, for an arbitrary fuel the experimental friction coefficient obtained from experiments with water can be expected to scale as ( $\mu$ is the dynamic viscosity)

$$
k_{l}=\sqrt{\frac{\rho_{l} \mu_{l}}{\rho_{\text {water }} \mu_{\text {water }}}} \cdot k_{\text {water }}
$$

It is also to be noted that the friction coefficient $k$ is not a material constant but linked to different variables in Eq. 11. This means that the friction coefficient may show some dependence upon length scale when extrapolating the results to very large tanks.

\section{Radiation and drainage models}

When the foam spread takes place for more than a couple of minutes the influence of the drainage and radiation-induced drainage becomes important and needs to be accounted for. A simple model for calculating the radiation from the burning fuel is used. The model is based on handbook formulas and correlations for calculation of flame heights, emissive power of the flames, and view factors. The flame diameter, which is the main input for calculation of the flame height, is set by the dimensions of the area not covered by foam in a pool. Empirical formulas are available for the average emissive power of large, sooty hydrocarbon fires [4]. These are developed for circular pool fires and suggest that emissive power decreases when the diameter increases.

An estimate of the flame height $h_{f}$ can be calculated by making use of the standard correlation formula for circular pool fires originally proposed by Heskestad [5],

$$
h_{f}=0.23 \cdot Q_{c}^{2 / 5}-1.02 \cdot D
$$

where $\dot{Q}_{c}$ is inserted in $(\mathrm{kW}), D(\mathrm{~m})$ is the diameter of the circular pool, giving $h_{f}$ in (m). This gives a good match between the observations and the calculated values of the flame height.

The foam loses weight during the foam spread. As mentioned above, in a fire situation there exist three sources of mass loss from the foam blanket: ordinary drainage, radiationinduced drainage, and evaporation. To more accurately model foam spread during longer foam spread times, sub models for these effects were included in the foam spread model. The ordinary drainage is a function of time, foam height, and type of foam. Several drainage experiments with different foam heights were performed to derive a empirical correlation. The radiation-induced drainage is a function of the same parameters, but of 
course also a function of the radiation. Similar empirical correlations were derived for this case. Studies of the evaporation $[6,7]$ have shown that the evaporation does not depend significantly on the type of foam, but mainly on the radiation. This assumption has been used in the model. More details on the mass loss experiments and correlations can be found in the report by Persson et al. [1].

\section{Numerical solution}

The set of equations governing the problem is highly non-linear and calls for a numerical solution. In the numerical solution scheme the total radial extension of the pool is divided into $N$ elements of equal size, in practise the length of each element $\Delta r$ is chosen to be between 1 to $5 \mathrm{~cm}$ depending upon the size of the pool and the time steps produced in the solution. Equation 5 is solved by making use of a fourth order Runge-Kutta algorithm starting from the leading edge, i.e. from the foam front and integrating backwards to the inlet. For each new time step $\Delta t$, the front is moved one step $\Delta r$ forward. For the new time step it is assumed that the density distribution from the previous time step is valid. The integration gives a new thickness distribution along the layer and at the inlet a new value of the thickness is produced. By integrating the new thickness distribution the total volume of foam is determined. Dividing this with the constant volume flow rate gives the total time since starting the release of the foam and thereby giving a new time step. Based on this new time step a new density distribution is calculated by integrating the mass loss due to drainage and evaporation along the foam layer according to Eq. 3 .

The approximation of a constant density distribution during the spatial integration means that there is a systematic error introduced into the solution. The error depends upon the length of the time step $\Delta t$. If this time step is deemed to be too long to introduce severe uncertainties due to the assumptions, the length of forward stepping of the front is decreased resulting in a shorter time step. Several runs with different forward length steps have been performed to ensure convergence.

\section{Model for gentle application at one or three points along the rim of a tank}

The model of foam spread with gentle application at the centre of a circular pool has been extended to cover the case with gentle application of foam at one or three different locations along the rim. To exploit the results available from the model for application at the centre, the case with foam release at the rim is assumed taking place at the centre of an imaginary pool with a diameter $D$ twice the diameter of the pool under consideration. Thus, $D=4 R_{p}$ where $R_{p}$ denotes the radius of the pool for which the calculation is to be performed. A schematic picture of the geometry and notations is displayed in Fig. 2.

The foam spread in the larger imaginary pool is assumed entirely symmetric (foam release at the centre of the imaginary pool) and the instantaneous location of the foam front is given by $R=R(t)$. The radial co-ordinate from the inlet on the rim is denoted by $r$. Friction against the sidewalls is neglected and the same correlations for friction are assumed to apply as for the boundary layer flow approximation used for the central inlet model. For this application it is advantageous to work with area coverage rather than giving the spread in terms of the extension $R$. Thus $A$ denotes the instantaneous area covered and $A_{p}$ the total pool area.

As the total area of the imaginary pool is 4 times $A_{p}$ one would expect that the proper volume flow rate would be $4 \dot{V}$. This would be the case if the foam layer had a uniform 
thickness over the pool. In reality the foam layer thickness decreases with the radius $r$. The real volume released when the pool is completely covered is less than $4 \dot{V}$. By integrating the theoretical foam layer profile it can be shown that the correct volume flow rate $\dot{V}_{i}$ for the imaginary pool is

$$
\dot{V}_{i}=16 \pi \dot{V} / 15
$$

If $R$ denotes the instantaneous foam extension in the imaginary pool, the instantaneous area covered with foam in the actual pool can be expressed

$$
\frac{A}{A_{p}}=1-\frac{2}{\pi}\left\{\left[1-2\left(\frac{R}{2 R_{p}}\right)^{2}\right] \arccos \left(\frac{R}{2 R_{p}}\right)+\frac{R}{2 R_{p}} \sqrt{1-2\left(\frac{R}{2 R_{p}}\right)^{2}}\right\}
$$

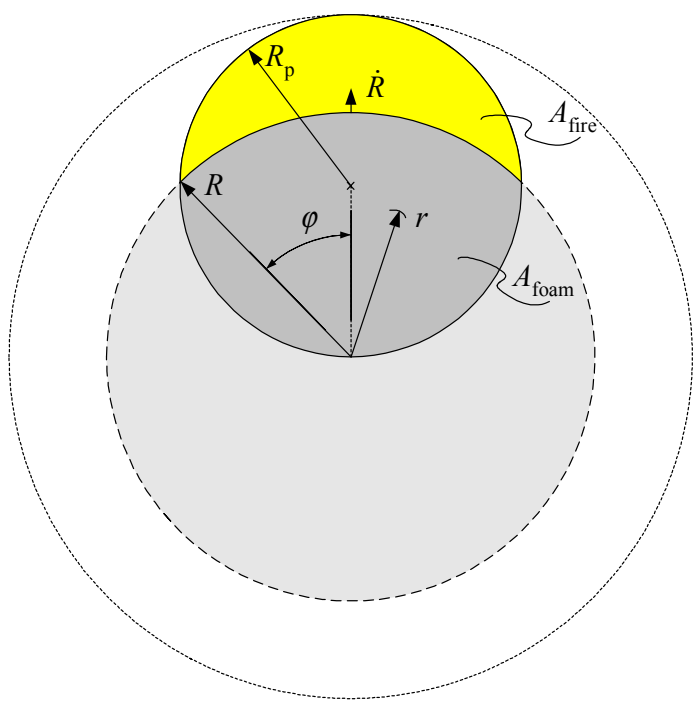

Fig. 2 Schematic of model for calculation of foam release at one location along the rim.

For the case with three inlets along the rim, the same model as for inlet at one point on the rim can be applied. Here it is assumed that the foam is released at three equally spaced points on the rim with a volume flow rate $\dot{V}_{i} / 3$ for each inlet. Each inlet point is assumed to spread the foam in an imaginary pool of size $D=4 R_{p}$. The instantaneous area is three times the area predicted by Eq. 15. In this approximate model no account is taken to the interaction between the three different foam layers spreading in the imaginary pools. It is expected that the model will be inaccurate at times when the foam fronts are approaching each other. 


\section{RESULTS AND DISCUSSION}

A large number of experiments have been conducted for determination of friction coefficients in the models and for generation of data for verification. In the experiments two types of foam were used, an AFFF-AR foam (AF for short) which is normally known to produce a "liquid" foam and a Fluoroprotein foam (FP) which is know to produce a more "stiff" foam. Most of the experiments were conducted with gentle application of the foam, i.e. where the foam was introduced via an application vessel to guarantee a smooth initial spread. In some experiments a semi-direct application was used where the foam was poured directly downwards towards the fuel surface without application vessel. More details about the experiments can be found in the report by Persson et al. [1]. In the following examples comparisons are presented between calculated and experimental results. In the calculations the accuracy has been controlled by performing several calculations with different length steps until there was a negligible change in the results.

\section{Experiments in a 1/2-symmetrical pool}

A number of experiments with foam spread on water in half symmetry have been performed in order to produce input data for the frictional resistance of the theoretical models. The radius of the pool was $10 \mathrm{~m}$. In the experiments the volume flow rate and the expansion ratio were varied.
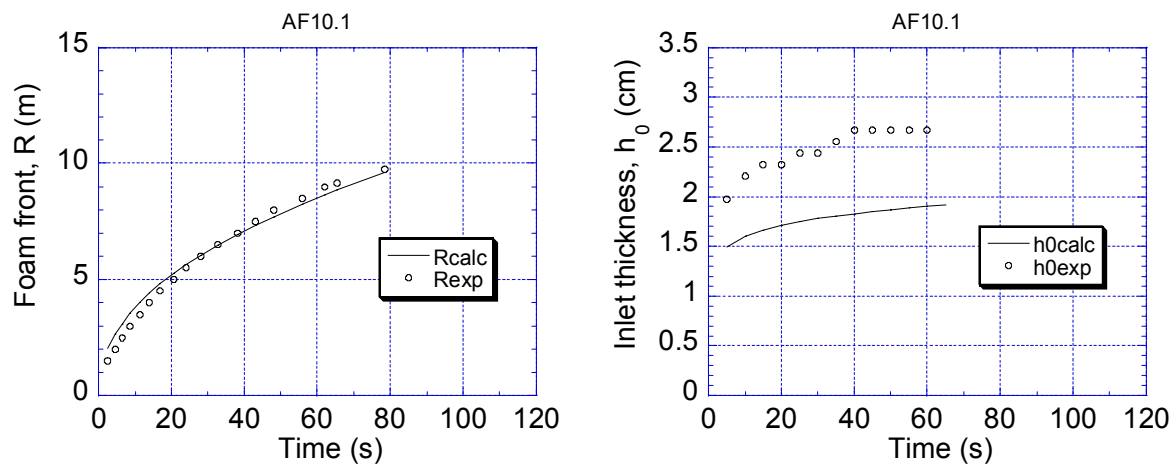

Fig. 3 Comparison between theory and experiments with AFFF-AR foam in half symmetry. The volume flow rate is $0.038 \mathrm{~m}^{3} / \mathrm{s}$, and expansion ratio 7.3 .

Figure 3 displays some typical results of foam front extension $(R)$ and inlet height of the foam layer $\left(h_{0}\right)$ for AFFF-AR foam. In the calculations the friction constant $k$ in Eq. 11 was set to 0.25 for the AFFF-AR foam. Similar results are obtained for FP foam with a friction constant 0.42 . The volume flow rate indicated in the Fig. 3 is valid for a full circular pool, i.e. twice the measured values in the experiments (half symmetry).

The general agreement between theory and experiments indicates that effects such as inertia and ordinary drainage are not important within the time span considered. The agreement between measured and calculated foam extension is better than for the inlet heights. The predicted shape of the foam layer is somewhat different from the measured, which causes discrepancies in the inlet height determination. A general trend is that the 
model underestimates the inlet thickness for the AF foam while the opposite is true for the FP foam.

\section{Gentle application at one point on the rim}

The agreement between theory and experiments is better for the FP foam than for the AF foam. The results from tests without an application vessel (Experiment 15.3 in Fig. 4) show a more rapid growth than when the foam is applied via a tray, especially for FP.

Similar results with good agreement have also been obtained for the case with gentle application at three locations along the rim.
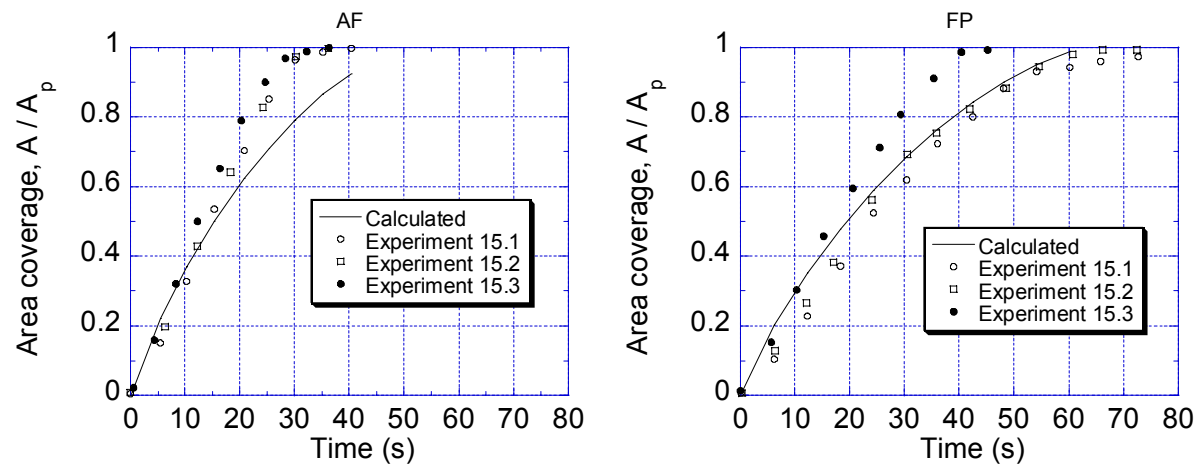

Fig. 4 Comparison between theory and experiments with AFFF-AR foam (left) and FP foam (right) applied at one location along the rim of a pool with diameter $4.7 \mathrm{~m}$. The volume flow rates are $0.0051 \mathrm{~m}^{3} / \mathrm{s}$ (left) and $0.0042 \mathrm{~m}^{3} / \mathrm{s}$ (right). Corresponding expansion ratios are 9.0 and 7.3 , respectively. The number 1 and 2 in the experiment "id" refer to experiments with application vessel (gentle application) while 3 refers to experiments without application vessel (semi-direct application).

\section{Tests in a large pool without fire}

Fig. 5 shows a comparison between theoretical foam flow and cold flow experiments on diesel oil for AFFF-AR and FP foams. In the case with AFFF-AR on diesel oil, a friction coefficient $k=0.52$ (corresponding to $\mathrm{k}=0.25$ from the experiments with water in the $1 / 2$-symmetry pool) has been used in accordance with Eq. 12. For FP the scaled friction factor according to Eq. 12 was $\mathrm{k}=0.88$. These results clearly verify the scaling of the friction coefficient.

In general the agreement is acceptable for both the AF and the FP foam. However, the friction factor is somewhat underestimated for the AFFF-AR foam. Still the uncertainty in area coverage is within $20 \%$. 

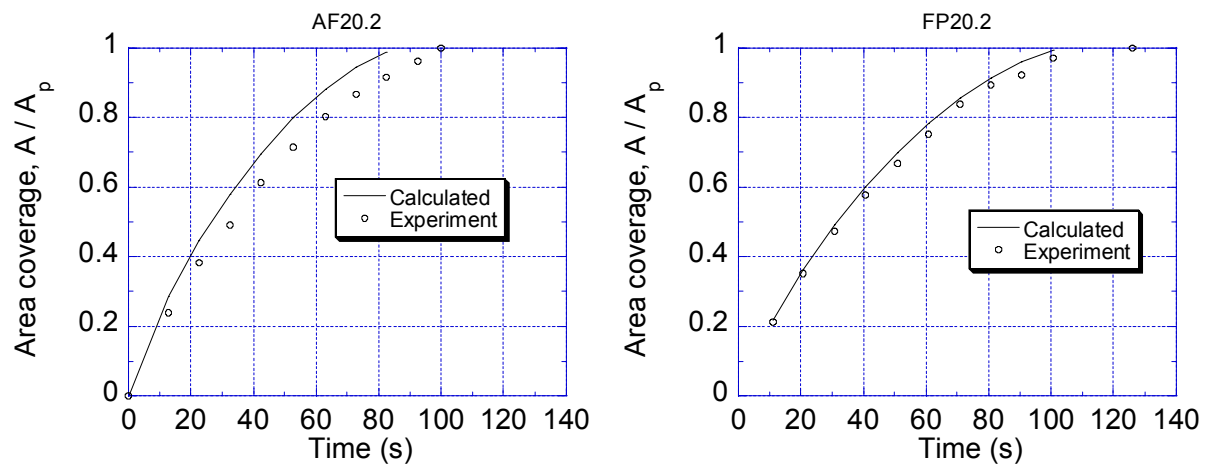

Fig. 5 Comparison between theory and cold flow experiments with gentle application of AFFF-AR (left) and FP foam (right) on diesel oil. Foam released at one location on the rim of a pool with diameter $13.4 \mathrm{~m}$. The volume flow rate is $0.047 \mathrm{~m}^{3} / \mathrm{s}$ with expansion ratio $6.6(A F)$ and $0.046 \mathrm{~m}^{3} / \mathrm{s}$ and $6.5(F P)$.

\section{Fire tests}

A few tests with fire have also been performed for verification of the theoretical model. In the calculations the friction shear stress used in cold flow has been reduced by approximately $20 \%$ to account for the decrease of the viscosity due to the heating up of the fuel.

For AFFF-AR there was probably some influence of foam destruction at the front for the lower volume flow rate, which slowed down the spreading (see Fig. 6). It should also be noted that the recordings of the foam coverage during the fire tests are more uncertain than in the cold tests.
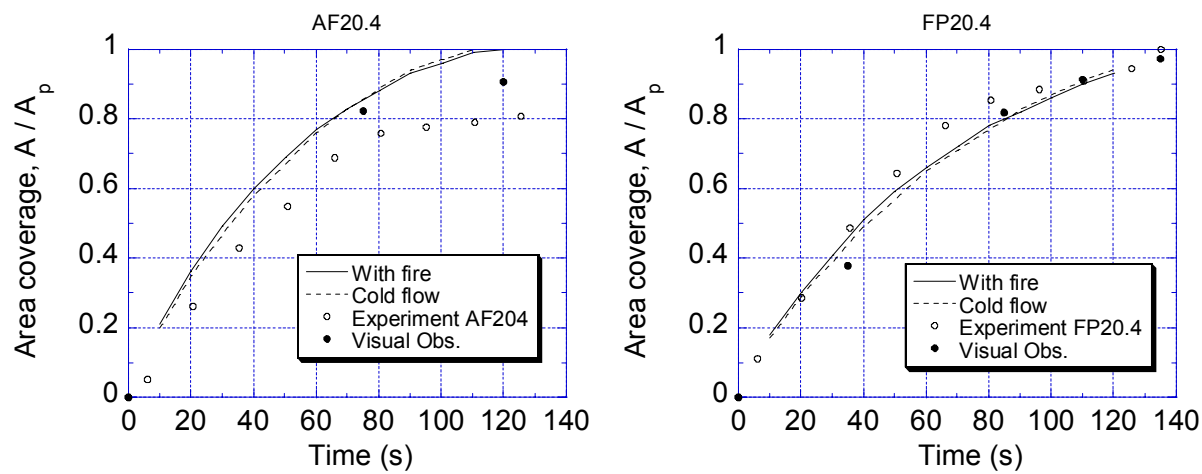

Fig. 6 Comparison between theory and fire experiments with gentle application of AFFF-AR foam (left) and FP foam (right) on diesel oil. Foam released at one location along the rim of a pool with diameter $13.4 \mathrm{~m}$. The volume flow rate is $0.037 \mathrm{~m}^{3} / \mathrm{s}$ with expansion ratio $7.9(\mathrm{AF})$ and $0.028 \mathrm{~m}^{3} / \mathrm{s}$ and $6.0(\mathrm{FP})$. 
As is seen from Fig. 6 the agreement is good for FP. The effect of the reduction in fuel viscosity, and possible changes in the internal properties of the foam, is obviously balanced by the influence of the drainage due to the fire and the spread with account taken to the fire is almost the same as for cold flow.

\section{CONCLUDING REMARKS}

The results can be summarised as follows:

- The agreement between calculated and experimental results is in general good for the laboratory scale data presented.

- The models presented are based on friction data from laboratory experiments with cold foam flow. An uncertainty in the models is how to scale the friction data when increasing the length scale by orders of magnitude (e.g. to tank diameter 100 - 120 $\mathrm{m})$. More work is needed to improve the accuracy of the friction data for larger tanks and for various types of foam.

- Further work is needed to incorporate the destruction of foam at the foam front, a phenomenon observed in the fire tests. This effect will be especially important when extending the models to large-scale tanks.

\section{REFERENCES}

[1] Persson B., Lönnermark A., Persson H., Mulligan D., Lancia A., and Demichaela M., "FOAMSPEX: Large Scale Foam Application - Modelling of Foam Spread and Extinguishment", SP REPORT 2001:13, 2001.

[2] Kroezen A.B.J., Groot Wassink J., and Schipper C.A.C., "The Flow Properties of Foam", Journal of the Society of Dyers and Colourists, 104, pp. 393-396, 1988.

[3] Persson B., Lönnermark A., and Persson H., "Modelling of Foam Spread in Channels with and without Fire", FOAMSPEX internal report no 37, SP Technical Note 2001:19, Borås, Sweden, 2001.

[4] Mudan K. S., and Croce P. A., "Fire Hazard Calculations for Large Open Hydrocarbon Fires", The SFPE Handbook of Fire Protection Enginnering, $\left(2^{\text {nd }}\right.$ ed), DiNenno P.J. (ed.), National Fire Protection Association, 1995.

[5] Drysdale D., An Introduction to Fire Dynamics, John Wiley \& Sons Ltd, Chichester, 1990.

[6] Isaksson S., and Persson H., "Fire Extinguishing Foam - Test Method for Heat Exposure Characterisation", SP REPORT 1997:09, Borås, Sweden, 1997.

[7] Persson H., "Fire Extinguishing Foams - Resistance Against Heat Radiation", SP REPORT 1992:54, Borås, Sweden, 1992. 